$26 \mid 2009$

Varia

\title{
La domus du centre hospitalier
}

Yvan Maligorne

\section{CpenEdition}

Journals

Édition électronique

URL : http://journals.openedition.org/rao/959

DOI : $10.4000 /$ rao.959

ISBN : 978-2-7535-1609-0

ISSN : 1775-3732

Éditeur

Presses universitaires de Rennes

Édition imprimée

Date de publication : 31 décembre 2009

Pagination : 318-323

ISBN : 978-2-7535-1086-9

ISSN : 0767-709X

\section{Référence électronique}

Yvan Maligorne, "La domus du centre hospitalier », Revue archéologique de l'Ouest [En ligne], 26 | 2009,

mis en ligne le 31 décembre 2011, consulté le 04 décembre 2020. URL : http://

journals.openedition.org/rao/959; DOI : https://doi.org/10.4000/rao.959 
observation avec des assemblages essentiellement modelés, des pâtes locales et des formes tronconiques à l'écart des formes sinueuses armoricaines. La surreprésentation des structures de stockage évoque une évolution de l'activité qui, d'une économie de subsistance, glisse vers une économie d'échange. Des relations avec les îles britanniques pour l'importation du lignite et avec les marges du Massif armoricain pour les meules en roche granitique témoignent de cette économie d'échange, mais c'est avec le territoire Lexovien que ces relations semblent les plus privilégiées avec l'approvisionnement en céramique glauconieuse et avec le monnayage qui est représenté par trois potins de la cité des Eburovices.

La seconde moitié du dernier siècle avant notre ère est témoin d'une relative désertion des sites de l'Étoile; les occupations sont pour cette période mal documentées, seules quelques céramiques laténiennes tardives et gallo-romaines précoces en sont les vestiges. Les fermes indigènes sont de nouveau occupées dans le courant du Ier siècle de notre ère; elles seront habitées jusqu'au milieu du second siècle et, pour le site II, jusqu'à la première moitié du IIIe siècle. Les sites I et III ne feront alors l'objet que d'occupations de faible densité, sous la forme principalement d'un puits et d'un four pour le site I et d'un puits, de quelques bâtiments, de fosses et de fossés pour le site III. Le site II est occupé plus densément avec l'installation de quatre édifices sur solins (un grand bâtiment de $18 \mathrm{~m}$ de côté à l'est et trois édicules à l'est); l'ensemble évoque le plan d'un sanctuaire.

Pour conclure, l'ouvrage de Chris-Cécile Vauterin est des plus intéressants. Si l'on peut regretter bien sûr que les vestiges du premier âge du Fer n'aient pas été présentés plus en détail dans le cadre de ce volume, on ne peut que saluer la qualité du travail mené sur les vestiges du second âge du Fer et de l'Antiquité. La monographie est complète, accompagnée de tout un cortège d'analyses proposant ainsi une vision interdisciplinaire de ces occupations. Les propositions de restitution des sites, eux-mêmes replacés dans leurs environnements, ouvrent sur de nouvelles problématiques qui seront confirmées ou infirmées à l'occasion des publications qui devraient suivre sur les sites voisins, dont celui d'Ifs fouillé en 2000 par E. Le Goff et son équipe. En fait, l'ouvrage de Chris-Cécile Besnard-Vauterin est la première pierre d'un édifice qui devrait, dans les prochaines années, nous offrir une vision renouvelée du second âge du Fer et du début de l'Antiquité en Plaine de Caen. Reste à attendre la suite!

David Giazzon (INRAP Basse-Normandie)

Le Cloirec, G. (dir.), 2008 - Carhaix antique. La domus du centre hospitalier. Contribution à l'histoire de Vorgium, chef-lieu de la cité des Osismes. Rennes, PUR, coll. "Documents archéologiques », 263 p., 205 fig., VIII pl. couleurs h-t. (ISBN 9782-7535-0671-8;25€).

L’ouvrage dirigé par Gaétan Le Cloirec constitue l'acte de naissance d'une nouvelle collection des Presses universitaires de Rennes, collection appelée à accueillir la publication des principales fouilles archéologiques conduites dans l'Ouest. Ce volume était très attendu, puisque la fouille dont il nous livre les résultats a eu lieu il y a près de treize ans, délai durant lequel on ne lui a consacré que quelques brèves notices, ce délai n'étant pas imputable à l'auteur, dont l'ouvrage était prêt depuis longtemps. Le travail a fait l'objet de quelques mises à jour, comme en témoignent des références ponctuelles à des travaux récents, mais pas d'une refonte globale, laquelle ne s'imposait d'ailleurs pas. On notera simplement qu'au moment de la rédaction, la fouille du centre hospitalier était la seule opération archéologique de grande ampleur conduite à Carhaix et qu'elle a maintenant été rejointe par la fouille de la « Réserve archéologique » de la rue du DocteurMenguy, dirigée par le même archéologue et dont on est en droit d'espérer que les résultats paraîtront dans des délais plus raisonnables.
L'ouvrage, remarquablement présenté, est clair et très abondamment illustré (on notera la présence de huit planches couleurs); les fautes d'orthographe et de syntaxe sont peu nombreuses. Au chapitre de l'illustration, on regrettera simplement l'absence de plans d'ensemble mêlant les différents états des bâtiments, qui auraient permis de mieux visualiser les évolutions parfois minimes intervenues entre les phases successives. Pour ce qui est du vocabulaire, on doit déplorer quelques imprécisions dans les termes architecturaux; ainsi, p. 48 , on nous parle - formulation pour le moins déroutante - d'un "portique appuyé sur une colonnade »; p. 122, sont évoquées des « colonnes rhodiennes " (alors que c'est un portique qui peut être qualifié de rhodien, supposant alors la mise en œuvre de supports adaptés; dans le cas qui nous occupe, les colonnes restituées par l'architecte n'ont rien de particulier, elles sont simplement juxtaposées); p. 122 encore, est signalé " le tétrastyle d'un atrium toscan " (alors que c'est le propre d'un atrium toscan que de ne pas comporter de colonnes). Ces difficultés auraient pu être aisé- 
ment levées par l'utilisation du Dictionnaire méthodique de René Ginouvès, outil de référence indispensable auquel le recours devrait désormais être systématique (Ginouvès 1992, 1998; Ginouvès, Martin 1985). Autre problème de forme, les renvois imprécis sous forme infra, supra, sans numéro de pages, qui rendent les vérifications longues et malaisées.

Après un premier chapitre qui rappelle les principales étapes de la recherche archéologique à Carhaix, situe la fouille dans son contexte topographique et présente les conditions de l'étude (en insistant sur les contraintes d'une fouille morcelée en trois secteurs entre lesquels il a été parfois impossible d'établir des relations stratigraphiques), l'ouvrage est structuré en trois parties : les occupations précédant la domus (chapitre 2); la domus tardive (chapitre 3), qui constitue (c'est apparent dès le titre du chapitre 2) le cœur de l'ouvrage; un élargissement du propos à l'ensemble de la ville de Carhaix (chapitre 4 et annexe), la fouille étant l'occasion de proposer un bilan renouvelé sur le chef-lieu des Osismes.

Le site du centre hospitalier occupe une position relativement périphérique dans le tissu urbain de la ville romaine de Vorgium; c'est sans doute ce qui explique que les premières traces d'occupation - si l'on excepte un souterrain de l'âge du Fer - ne remontent qu'à la seconde moitié du $\mathrm{I}^{\text {er }}$ siècle. Mais, précision importante, elles s'inscrivent dans un cadre dont la mise en place est plus précoce. Réparties de part et d'autre d'une voie cardinale appelée à structurer le site durant toute l'Antiquité, ces constructions de terre et de bois sont encore modestes, mais respectent les grandes orientations de la trame urbaine; la pratique d'activités artisanales est probable. Trois îlots peuvent d'ores et déjà être distingués, séparés par le cardo et par un decumanus secondaire. L'occupation gagne en densité et en importance à partir de la fin du I $^{\text {er }}$ siècle et dans le courant du IIe. Les bâtiments font désormais un large usage de la maçonnerie et subissent des réfections régulières. Ils obéissent à une typologie variée, depuis la construction la plus modeste jusqu'à la domus parfaitement caractérisée; en cela, le site de l'hôpital offre une manière de condensé des types de maisons et d'édifices commerciaux que l'on connaissait déjà dans l'Ouest, dispersés entre différents sites de Corseul, Rennes ou Jublains.

Les activités artisanales disparaissent progressivement, G. Le Cloirec précisant qu'il n'en reste plus de traces à la fin $\mathrm{du}$ IIe s.; l'occupation a dorénavant une fonction résidentielle et commerciale. Deux maisons retiennent particulièrement l'attention. Le « bâtiment I » présente deux ailes (mais une troisième a peut-être disparu) précédées de portiques et trahit sans nul doute une influence de la domus à péristyle, sans que son propriétaire ait eu les moyens de la doter de tous les caractères de la maison de notable. Comme le relève justement G. Le Cloirec, cette maison rappelle celle du Champ-Mulon à Corseul; que l'édifice carhaisien ait comporté deux ou trois ailes, le même esprit a présidé à la conception des deux maisons. C'est en tout cas de façon un peu abusive, croyons-nous, que Pascal Vipard range dans une recherche récente la maison du Champ-Mulon parmi les domus de notables (Vipard, 2007). Ce qui importe en l'espèce n'est pas la présence de seulement trois galeries dans le portique (on connaît nombre de péristyles incomplets), mais le fait que l'espace défini par le porticus triplex ne soit pas fermé sur le quatrième côté, observation qui l'empêche de prétendre au titre valorisant et sociologiquement signifiant de péristyle. Le "bâtiment $\mathrm{D}$ » (p. 50), malheureusement fouillé de manière partielle, mérite en revanche le titre de domus. On notera que l'orientation du péristyle y implique plutôt une entrée vers le nord, dans l'hypothèse (très généralement vérifiée) d'une disposition axiale des principales composantes de la demeure, ce qui suppose donc une ouverture sur une voie secondaire. D'ailleurs, dans l'hypothèse où les structures enregistrées comme "bâtiment $\mathrm{C}$ " appartiendraient à la même demeure, on remarque qu'elles ne comportent rien qui soit identifiable à un ensemble vestibule-fauces. Les fouilles de la Réserve archéologique ayant révélé les substructions de deux domus sévériennes, toutes deux dotées d'un péristyle incomplet et d'un balneum, force est de constater que Carhaix fournit à elle seule la moitié des " maisons nobles" connues en Armorique durant le Haut-Empire.

Après une phase de transition à la fin du II siècle, la période sévérienne est marquée par un essor du quartier : plusieurs édifices font l'objet de réfections importantes (les bâtiments D' et J), un autre est élevé ex novo (le bâtiment L). La bonne santé de l'agglomération en ce début du $\mathrm{III}^{\mathrm{e}}$ siècle est amplement confirmée par les fouilles de la Réserve archéologique.

Le chapitre 3 est tout entier consacré à l'étude d'une grande domus. Ce chapitre est le plus intéressant et associe l'archéologue à un architecte, Vincenzo Mutarelli, à qui l'on doit déjà l'étude architecturale du quartier de Monterfil II à Corseul (Kerébel, 2001) et celle, encore largement inédite, du temple du Haut-Bécherel dans la même commune (Provost et al., à paraitre). Cette étape se décompose en trois parties : présentation des vestiges, restitution architecturale et analyse fonctionnelle, auxquelles il faut ajouter des études de mobiliers.

Cette domus est constituée dans l'îlot III par la réunion de deux demeures déjà existantes, les bâtiments I et L. Ils sont reliés par un mur précédé d'un porche monumental et un péristyle assure l'unification de la demeure. Si l'on peut discuter des termes les mieux adaptés pour désigner les demeures des phases précédentes, le terme de domus, avec toutes ses implications morphologiques et sociologiques, est parfaitement adapté à cette maison. Les indices chronologiques sont minces, mais assurent toutefois que la demeure 
n'est pas antérieure à la fin du IIIe s.; elle date même plus probablement de la période constantinienne, ce qui en fait assurément la domus la plus tardive identifiée dans l'Ouest.

Les auteurs insistent avec raison sur l'habileté des bâtisseurs; ceux-ci ont construit le péristyle qui en occupe le cœur à partir d'un carré idéal dont les dimensions sont déterminées par la volonté d'assurer à la salle de réception s'ouvrant au centre de l'aile nord une position rigoureusement axiale. Si l'on ne peut que les suivre, il faut aussitôt préciser que les ambitions du concepteur du projet ont été servies par la position relative des deux bâtiments initiaux, elle-même conséquence du découpage parcellaire du site : en effet, du nord au sud, la distance entre la façade de l'ancien bâtiment $\mathrm{L}$ et l'angle interne du portique du bâtiment I (donnée que l'architecte ne pouvait modifier) est identique à la distance qui sépare l'extrémité orientale du bâtiment $\mathrm{L}$ du mur construit pour relier les deux bâtiments. On se rend d'ailleurs compte au fil de l'analyse architecturale que ce péristyle n'est qu'un "bricolage ", astucieux certes, mais imparfait. Il présente une inflexion près de l'angle nordouest où, de surcroît, si l'on en croit les propositions formulées, les colonnes laissent place à un mur plein; surtout, les colonnades ne présentent pas partout un rythme identique. Le péristyle est donc incomplet et pâtit d'irrégularités rythmiques, ce en quoi il ne mérite pas le titre de quadriportique, sa valeur de structure d'intégration et d'unification s'en trouvant sérieusement amoindrie.

Cette ample demeure fait l'objet d'une tentative de restitution, partie la plus novatrice de l'ouvrage. Les réflexions conduites par l'architecte portent avant tout sur les ailes septentrionale et occidentale de la maison, seules dégagées, tandis que les ailes orientale et méridionale - en l'espèce les portiques qui les précèdent - ne sont évoquées que rapidement. Nous ne pouvons discuter en détail d'une restitution qui s'appuie avant tout sur une analyse métrologique des vestiges, qu'elle prolonge le cas échéant par une étude des quelques blocs d'architecture retrouvés lors de la fouille (et dont l'appartenance à la domus n'est d'ailleurs pas assurée). Nous nous contenterons de quelques remarques.

1) Relevons d'abord que Vincenzo Mutarelli fait largement appel à la juxtaposition de colonnes de diamètres et de hauteurs différentes, système qu'il avait déjà imaginé pour la restitution du grand bâtiment commercial de Monterfil II à Corseul. Le rôle d'articulation de systèmes architecturaux différents qu'il leur assigne (par exemple l'articulation d'un portique rhodien) serait mieux rempli par des « colonnes bilobées ", associant au sein d'un même support deux colonnes de diamètres et hauteurs différents, ou encore par de nombreux types de supports complexes, qui ont l'avantage d'être parfaitement attestés dans notre région (Maligorne 2006, p. 113-114).
2) Penchons-nous maintenant sur la restitution d'un portique à étage en façade de l'aile nord. La présence d'un étage est prouvée par l'existence de deux escaliers mais le second portique est sans doute justifié un peu rapidement, alors même que cette structure revêt un caractère exceptionnel. Nous croyons percevoir ici un parti pris privilégiant systématiquement pour les élévations les solutions les plus monumentales, les plus complexes et les plus valorisantes, au motif - explicitement formulé à plusieurs reprises - que la planimétrie désigne un projet de qualité. Peut-être aurait-il été plus prudent et méthodologiquement moins hasardeux de proposer deux solutions.

3) Le centre de l'aile nord est occupé par une remarquable salle d'apparat; elle fait l'objet d'une étude planimétrique et volumétrique très solide, qui emporte totalement l'adhésion. Mais la restitution de ses ordonnances internes n'est pas aussi convaincante. La présence de deux paires de colonnes dans la salle 10 (en fait la partie antérieure de la salle d'apparat) ne nous paraît pas pleinement assurée : les quatre empreintes quadrangulaires préservées dans le dallage en opus sectile peuvent correspondre à des motifs comparables à ceux qui flanquent les angles de l'emblema. Au passage, on a quelque peine à comprendre pourquoi il est fait longuement mention de colonnes dont aucun fragment n'a été identifié, alors que rien ou presque n'est dit de blocs d'entablement en calcaire retrouvés par les fouilles et dont l'appartenance à cet espace est hautement probable. Un fragment portant de vagues traces de rinceau ne se prête pas à des commentaires très développés - si ce n'est que ce type de décor est tout à fait exceptionnel dans nos régions, ce qui accroît encore la richesse de la salle d'apparat - mais un important fragment de corniche ionique muni d'un soffite curviligne non retenu sur la planche réunissant les fragments du décor architectonique - méritait à l'évidence plus que de simples allusions.

4) On a parfois l'impression que les solutions proposées ne procèdent pas de l'analyse d'un dossier comparatif établi selon des critères contraignants, mais que les demeures les mieux connues du monde romain (domus campaniennes et africaines essentiellement) offrent un répertoire de formes dans lequel l'architecte se sent autorisé à puiser pour justifier les propositions les plus hardies. Le meilleur exemple nous est fourni par les développements consacrés à l'entrée de la domus, partie la plus contestable de la restitution. Les vestiges archéologiques sont très ténus : des lambeaux de sol en béton attestent la présence d'un espace couvert, devant lequel quatre taches de mortier matérialisent l'emplacement de probables supports verticaux. À partir de ces données lacunaires, V. Mutarelli restitue un portique pour le moins étonnant : très haut, il est composé de piliers doriques soutenant un entablement comportant une frise de métopes 
et triglyphes. En guise de parallèles, sont invoqués deux exemples : la maison pompéienne de Iulia Felix et la salle des Pilastres doriques de la villa Hadriana. Mais dans aucun de ces exemples les piliers n'ornent une entrée : ils jouxtent un jardin dans le premier cas et ornent un espace dont la destination n'est pas assurée dans le second. On remarquera encore, constat dont on aurait tort de ne tenir aucun compte, qu'aucun pilier n'est connu, même sous une forme fragmentaire, dans l'ouest de la Gaule : sont seulement mis en œuvre des colonnes, des pilastres (en petit nombre et presque toujours engagés dans l'angle d'une structure) ou des supports composites (Maligorne 2006, p. 113-115). Objection plus sérieuse encore, l'ordre dorique souffre dans la partie occidentale de l'Empire d'une désaffection presque totale depuis le tout début de la période julio-claudienne. Ses occurrences postérieures sont absolument exceptionnelles et se justifient par un système de références savantes qui ne s'expliquent que dans des ambiances très particulières : ainsi, le recours à cet ordre au $\mathrm{II}^{\mathrm{e}}$ siècle pour le temple d'Esculape de Lambèse ne peut se comprendre que comme une référence aux asklepieia de Grèce, dont celui d'Épidaure occupe le premier rang (Janon 1985, p. 84-85); quant à l'apparition de cet ordre dans la villa impériale de Tivoli (dans la salle des Pilastres doriques, mais encore sur le Temple de Vénus), elle s'explique par la vocation proprement anthologique d'un complexe qui entendait signifier l'unité de l'Empire sous l'égide de la culture grecque en offrant un catalogue complet de formes (Gros 2001, p. 369-370). Si l'on en vient à la hauteur de ce portique, on se rend compte qu'elle n'est justifiée que par des arguments esthétiques qui relèvent de ce parti pris de monumentalité évoqué plus haut. L'analyse des vestiges conduit certes à imaginer un portique, mais la constitution d'un dossier comparatif raisonné aurait montré que ces portiques étaient très généralement composés de colonnes; quant à la prise en compte de la dimension chronologique (l'architecture a une histoire), elle aurait conduit, croyons-nous, à exclure absolument le recours à l'ordre dorique, tant on peut douter que les lapidarii gaulois du début du $\mathrm{IV}^{\mathrm{e}}$ siècle étaient encore familiers du problème de la contraction angulaire.

5) Une dernière remarque, qui ne porte pas à conséquence sur la restitution des volumes et n'affecte que des points de détail. Il nous semble que les propositions ne tiennent pas suffisamment compte des données régionales : Vicenzo Mutarelli restitue par exemple systématiquement des colonnes toscanes munies d'une base attique. La solution serait pertinente dans la plupart des régions du monde romain occidental, mais il se trouve que les colonnes toscanes de la région reposent le plus souvent sur des bases toscanes, à tore unique. L'explication la plus probable réside dans le matériau, le granite étant plus dur à travailler que le calcaire; il n'est pas indifférent de relever que le décor architectonique de la domus a fait appel au granite - au moins pour les ordonnances extérieures - et que la seule base retrouvée sur le site est toscane.

Ces réserves n'enlèvent rien à la qualité de la tentative de restitution. Cette entreprise ardue, devant laquelle beaucoup auraient renoncé, est exposée avec clarté et soutenue par une illustration abondante et toujours pertinente, qui fait fort heureusement une place de choix aux éléments de comparaison invoqués, ce qui permet au lecteur de suivre aisément l'argumentation. Si quelques points paraissent contestables ou mal assurés, si quelques hypothèses alternatives auraient mérité d'être explorées plus avant, la validité de la démarche d'ensemble est démontrée par les indéniables correspondances que fait apparaitre l'analyse modulaire.

L'analyse planimétrique et la restitution des élévations permettent à Gaétan Le Cloirec de formuler quelques hypothèses sur la fonction des différents espaces. Ici encore, l'argumentation est clairement exposée et les propositions apparaissent cohérentes. Nous regretterons cependant que l'hypothèse qui veut reconnaître un triclinium dans la salle orientale de l'aile nord ne fasse l'objet d'aucun développement.

Mais ce que l'on retiendra surtout de cette partie, c'est la remarquable étude de la salle d'apparat de l'aile nord, qui se trouve replacée dans l'évolution des salles de réception en contexte domestique. L'auteur démontre que la forme cruciforme de cet espace trouve sa place dans des séries de l'Antiquité tardive. Il est à ce propos un peu dommage de devoir attendre la conclusion pour que soit cité le meilleur parallèle régional, à savoir le triconque de la villa de Kerran en Arradon. Le parallèle invoqué avec la forme en croix d'un atrium à alae prolongé par un tablinum est effectivement saisissant, mais il a toutes chances d'être purement formel puisque l'atrium a depuis longtemps été délaissé par l'architecture domestique urbaine. Les réflexions sur la valeur plurifonctionnelle de cet espace, dans lequel on peut reconnaître à la fois une salle de réception pour la cérémonie du banquet et une salle d'audience, emportent l'adhésion. Cet espace d'apparat (le terme est suffisamment neutre pour recouvrir ces différentes fonctions) pourrait donc dans la première fonction accueillir des pairs du dominus, dans la seconde des inférieurs et des dépendants.

Toutes les réflexions sur la typologie de la maison et de ses différentes composantes appelaient, croyons-nous, une réflexion sur le statut de ce dominus. Or, si l'on relève quelques remarques isolées et quelques allusions à ces problèmes, la question n'est jamais abordée de front, sans doute par excès de prudence. Dans un article récent, Pascal Vipard propose de reconnaître dans les domus à péristyle du Haut-Empire les demeures des décurions composant l'ordo des cités; il 
donne ainsi un contenu plus précis au terme de " notables " traditionnellement retenu pour désigner les propriétaires de ces maisons (Vipard 2007, p. 229-233). La proposition est très séduisante, même si elle a sans doute un caractère trop systématique : d'abord parce qu'elle pose avec acuité le problème des domus situées dans des agglomérations secondaires, comme, dans l'Ouest, à Rezé, ensuite parce qu'on ne voit pas qu'un interdit systématique ait frappé les personnages qui n'appartenaient pas à l'ordo, leur enjoignant de ne pas doter leur maison urbaine des composantes obligées de la domus. Dans le cas présent, pourtant, l'hypothèse d'une appartenance du dominus à l'ordo decurionum des Osismes nous semble absolument incontournable : nous avons très probablement affaire à l'un de ceux que l'on nomme désormais du titre de curiale et qui devaient posséder une résidence urbaine, première garantie d'une participation effective aux affaires de la communauté civique. La constitution de la maison correspond peut-être à son accession à ce statut. Il a choisi une typologie rappelant la domus du Haut-Empire, mais avec une différence notable sur laquelle la publication, étrangement, ne dit mot : si l'on retrouve le triptyque entréepéristyle-salle d'apparat, la rupture est radicale avec l'axialité qui auparavant unissait ces trois espaces en une séquence cohérente, permettant au visiteur de saisir, dès le seuil de la maison, ses principales articulations.

L'étude de la domus est complétée par trois études de mobilier. Françoise Labaune a pris en charge le verre (p. 143-151), ses analyses confirmant l'occupation tardive de la domus et l'opulence de ses occupants. Les bois travaillés sont étudiés par Anne Dietrich (p. 151-158), la découverte d'un seau intact au fond du puits méritant une mention particulière. Le mobilier en cuir est examiné par Véronique Montembault (p. 158-161).

La fin de la domus et des constructions environnantes fait l'objet de développements précis et étayés (p. 162-171). Ce que l'auteur appelle "l'occupation conventionnelle " de la domus se prolonge jusqu'au milieu du $\mathrm{IV}^{\mathrm{e}}$ siècle, après quoi l'occupation change de nature, les lieux se dégradant rapidement avant que les matériaux ne soient récupérés. L'îlot I pourrait avoir connu un abandon plus précoce et rien, en tout cas, ne permet de supposer un abandon brutal affectant simultanément l'ensemble du quartier.

Le dernier chapitre marque un élargissement du propos à l'ensemble de la ville de Carhaix : la première fouille de grande ampleur qui y ait été pratiquée donne à Gaétan Le Cloirec l'occasion d'un bilan sur la ville et sa place dans la civitas des Osismes. La démarche est heureusement complétée et étayée par un inventaire des sites archéologiques de Carhaix; pour mesurer la richesse et l'utilité de cet instrument de travail, il n'est que le comparer aux pages que
Patrick Galliou consacre à la ville dans son volume de la Carte archéologique de la Gaule (Galliou 1989).

Ce bilan renouvelé fournit des informations plus solides sur la superficie de la ville, que l'auteur évalue à 90 ha au moins, précisant qu'elle pouvait atteindre 130 ha, et sur sa structure : l'existence d'un carroyage orthogonal, jusqu'alors simplement pressentie, est désormais parfaitement établie et Gaétan Le Cloirec expose des hypothèses stimulantes sur les principes qui en auraient régi la formation. Carhaix n'en reste pas moins une ville mal connue : à l'exception des aqueducs, qui ont fait l'objet d'une étude détaillée de la part d'Alain Provost et Bernard Leprêtre, on ne sait pas grand-chose des monuments publics. Les blocs et fragments d'architecture auraient cependant permis d'étoffer quelque peu ces développements, puisqu'ils offrent accès à tout un pan de la parure monumentale.

L'auteur revient sur le statut de Carhaix au sein de la cité des Osismes. Sans doute ces développements n’apportent-ils pas de grandes nouveautés, la plupart des questions ici abordées ayant déjà été réglées par L. Pape dans sa thèse (Pape 1978, p. 31-36) : Vorgium était bien le chef-lieu de la civitas, quand Vorganium était une agglomération secondaire, qu'il faut sans nul doute identifier à Kérilien-Coatalec. Le géologue Louis Chauris a d'ailleurs démontré que le milliaire de Kerscao, qui mentionne Vorganium comme caput viae (agglomération par ailleurs mentionnée par Ptolémée) avait été taillé dans un affleurement strictement local; la borne n'a donc pas été déplacée et peut être légitimement invoquée pour localiser cette ville. Le problème du statut de Vorgium dans l'Antiquité tardive, déjà évoqué dans le premier chapitre, fait l'objet de quelques remarques. Il faut rappeler que l'hésitation n'est pas de mise et que le maintien de la dignité de chef-lieu n'est pas contestable puisque la Notitia Galliarum, qui énumère des villes et non des cités, en apporte la preuve jusqu'au tournant des ive et $\mathrm{v}^{\mathrm{e}}$ siècle (Maligorne 2004, p. 62-64). Si la découverte de la domus du centre hospitalier est parfaitement cohérente dans ce contexte, l'archéologie n'est pas en mesure d'apporter à elle seule des réponses définitives à ces problèmes institutionnels : on le voit bien avec la civitas des Coriosolites où Alet connait une occupation tardive plus importante que Corseul, laquelle reste pourtant chef-lieu.

La structure du livre de Gaétan Le Cloirec n'est pas sans rappeler celle de l'ouvrage qu'Hervé Kerébel a consacré aux fouilles du secteur de Monterfil II à Corseul; comme son prédécesseur, ce volume fait progresser de façon significative notre connaissance d'un chef-lieu de l'Ouest et est appelé à constituer un outil indispensable à qui s'intéresse à l'urbanisation de l'Armorique. 


\section{Bibliographie}

Galliou, P., 1989 - Carte archéologique de la Gaule. Le Finistère (29), Paris, Académie des Inscriptions et BellesLettres, $229 \mathrm{p}$.

Ginouvès, R., 1992 - Dictionnaire méthodique de l'architecture grecque et romaine. II. Eléments constructifs : supports, couvertures, aménagements intérieurs, Rome, Athènes, Bibliothèque des Écoles françaises de Rome et d'Athènes, 347 p., 90 pl.

Ginouvès, R., 1998 - Dictionnaire méthodique de l'architecture grecque et romaine. III. Espaces architecturaux, bâtiments et ensembles (CEFR 84), Rome, Athènes, Bibliothèque des Écoles françaises de Rome et d'Athènes, 352 p., 115 pl.

Ginouvès, R. et Martin, R., 1985 - Dictionnaire méthodique de l'architecture grecque et romaine. I. Matériaux et techniques, Rome, Athènes, Bibliothèque des Écoles françaises de Rome et d'Athènes, 303 p., 65 pl.

Gros, P., 2001 - L'architecture romaine, 2, Maisons, palais, villas et tombeaux, Paris, Picard, 527 p.
JANON, M., 1985 - Recherches à Lambèse III : essais sur le temple d'Esculape, Antiquités africaines, 21, p. 35-102.

KeréBel, H. (dir.), 2001 - Corseul (Côtes-d'Armor), un quartier de la ville antique, les fouilles de Monterfil II. Paris, Maison des Sciences de l'Homme (DAF 88), 249 p.

Maligorne, Y., 2004 - Carhaix et Corseul : deux « capitales éphémères »? Brèves considérations sur une hypothèse mal fondée, Bulletin de la Société archéologique du Finistère, 133, p. 61-67.

MALIgORNe, Y., 2006 - L'architecture romaine dans l'ouest de la Gaule, Rennes, PUR, 229 p.

Pape, L., 1978 - La civitas des Osismes à l'époque galloromaine, Paris, Klincksieck, 243 p., annexes 296 p., 16 pl. h-t.

Provost A., Mutarelli V., Maligorne Y., à paraître - Le Haut-Bécherel à Corseul, sanctuaire public des Coriosolites (à paraître aux PUR).

VIPARD, P. , 1997 - Maison à péristyle et élites urbaines en Gaule sous l'Empire, Gallia, 64, p. 227-277.

Merdrignac, B., 2009 - La Bretagne des origines à nos jours, Rennes, éditions Ouest-France, 127 p. (ISBN 978-2-73734496-1; 15,90€).

C’est dans la série « Histoire des provinces » que Bernard Merdrignac, professeur d'Histoire médiévale, vient de publier, en 2009, une histoire de la Bretagne, des origines au XXI ${ }^{\mathrm{e}}$ siècle. Spécialiste du Premier Moyen Âge et de l'hagiographie bretonne, B. Merdrignac, a enseigné à Rennes 2 pendant près de trente années et rien de ce qui touche à la recherche historique dans l'Ouest ne lui est étranger. Rien d'étonnant donc à ce que sa plume ait été sollicitée pour écrire ce volume.

L'exercice qui consiste à vulgariser la recherche n'est pas très souvent pratiqué par les universitaires et chercheurs, plus habitués à dialoguer entre eux via les colloques, les revues et les ouvrages spécialisés. Sans doute était-ce une gageure de synthétiser en 126 pages, sans notes infra-paginales et en laissant de surcroît la place à une riche iconographie, un sujet aussi riche, mais cloisonné en des centaines d'articles approfondis, traités par les spécialistes de chaque période qui ne portent presque jamais un regard global sur le temps long. Et c'est pourtant là un exercice absolument nécessaire si l'on veut que le public en général soit informé des avancées archéologiques et historiques les plus récentes et qu'il ne tienne pas pour intangibles des données vieilles parfois de plus d'un siècle.

Pour la Préhistoire, la Protohistoire et l'Antiquité, l'ouvrage fait état des découvertes les plus importantes, telles celles du site paléolithique de Ménez-Dregan, ou du grand site de l'âge du Fer de Paule, en les resituant sur fond de découvertes anciennes et dans le contexte général européen. Bien sûr, les archéologues regretteront qu'on n'ait pas fait état des innombrables sites anciens découverts en prospection aérienne depuis trente ans et qui renouvellent totalement l'image des campagnes de l'Ouest avant la conquête romaine; les philologues discuteront l'affirmation selon laquelle la langue celtique aurait partout été la même en Europe et les historiens penseront que l'accent n'a pas été suffisamment mis sur les massacres commis par les conquérants romains, par exemple chez les Vénètes. C'est donc avec une petite frustration qu'ils constateront que, comme dans la plupart des ouvrages de ce type, l'archéologie, et donc l'histoire des 500000 ans antérieurs à l'an 500, en sont réduits à 21 pages sur 126 . Ils regretteront un peu que l'archéologie, ne soit traitée que comme préambule à l'Histoire, alors qu'elle est constitutive de la matière même de celle-ci.

Pour le haut Moyen Âge, sa période de prédilection, on ne pouvait attendre de Bernard Merdrignac que d'intéressants développements; on pense entre autres aux lignes novatrices consacrées aux royaumes doubles de Grande et de Petite Bretagne. Nombre de pages traitent de l'histoire de la Bretagne médiévale en faisant le point sur les études les 